\title{
Versorgungsmonitoring mit Routinedaten am Beispiel Diabetes mellitus
}

\section{Kontext \\ $\checkmark$}

Das Bemühen um Versorgungsqualität erfordert eine Transparenz über den Versorgungsalltag. Wünschenswert sind hierzu Verfahren, die zeitnah, kontinuierlich und kostengünstig Steuerungsinformationen bereitstellen. Eine relevante Datenquelle sind die Daten der Gesetzlichen Krankenversicherung (GVK) [1]. Am Beispiel des Diabetes mellitus soll aufgezeigt werden, welche Kennziffern und Qualitätsindikatoren sich routinemäßig z.B. für eine Gesundheitsberichterstattung als individuelles Feedback an Ärzte/Netze oder zur Evaluation von Interventionen auf der Basis von Daten der GKV abbilden lassen.

\section{Material/Methode \\ $\nabla$}

Datengrundlage ist die Versichertenstichprobe AOK Hessen/KV Hessen, eine 18,75\%ige Zufallsstichprobe von Versicherten der AOK Hessen, mit dem Beobachtungszeitraum von 2000 bis 2009. Zur Definition eines Patienten mit Diabeteserkrankung wurden verschiedene Kriterien (Krankenhaus-Entlassungsdiagnose, ambulante Diagnose, Arzneimittel, Blutzuckermessung bei Einmalnennung einer Diagnose) herangezogen $[2,3]$. Die Indikatoren wurden aus LeitlinienEmpfehlungen abgeleitet. Ambulante ärztliche Leistungen wurden mittels Ziffern des Einheitlichen Bewertungsmaßstabs (EBM) und stationäre Operationen mittels Operationsschlüssel (OPS) für die jeweiligen Jahre erfasst.

\section{Ergebnisse \\ $\checkmark$}

Standardisiert auf die Alters- und Geschlechtsverteilung der deutschen Wohnbevölkerung (Stichtag 31. Dezember des Vorjahres) stieg die Prävalenz des behandelten Diabetes von 6,5\% im Jahr 2000 auf 9,7\% im Jahr 2009 (+ 49\%; Anstieg aufgrund der Alterung: + 14\%). Die Behandlungsprävalenz mit Metformin bei Patienten mit oraler Antidiabetika-Therapie zeigt eine deutliche Zunahme (2000: 48\%, 2009: 79\%) bei Rückgang des Stellenwertes der Sulfonylharnstoffe (2000: 48\%, 2009: 10\%). Relevante Untersuchungen, wie z.B. die Überprüfung der Blutzuckerwerte, der Blutfettwerte und der Nierenfunktion, wurden im Jahr 2008 zwar deutlich häufiger als im Jahr 2000 durchgeführt, allerdings noch nicht bei allen Patienten dokumentiert (Daten 2009 nicht darstellbar wegen veränderter Vergütung). Augenhintergrund-Untersuchungen sind seit 2003 bei ca. einem Drittel der Patienten mit Diabeteserkrankung dokumentiert. Eine deutliche $\mathrm{Zu}-$ nahme zeigt sich bei der Verordnung von Statinen zur kardiovaskulären Prävention bei den Patienten mit dokumentierter Fettstoffwechselstörung (2000: 32\%, 2009: 54\%). Als Ergebnisparameter können stationäre Aufenthalte mit der Entlassungsdiagnose Diabetes mellitus und Amputationshäufigkeit herangezogen werden. Für Letzteres war unseren Daten zufolge im Jahr 2000 bei 6,0 Promille der Patienten mit Diabeteserkrankung eine Amputation der unteren Extremitäten und Füße dokumentiert (2009: 4,7 Promille).

\section{Schlussfolgerung}

$\nabla$

Mittels eines Versorgungsmonitoring auf der Basis von GKV-Daten kann kontinuierlich dargestellt werden, in welchem Umfang bestimmte Therapie-Empfehlungen und Verlaufskontrollen durchgeführt werden und wie sich Ereignishäufigkeiten im zeitlichen Verlauf entwickeln. Die Kennziffern können für unterschiedliche Patientengruppen (Alter, Geschlecht, Komorbidität) erhoben werden, um Hinweise auf besonderen Versorgungsbedarf zu erhalten. Ebenso ist ein Monitoring der Kosten möglich [3]. Kritisch zu prüfen ist, welche Kennziffern sich als Qualitätsindikatoren eignen, d.h., ob sie eine Steuerungswirkung entfalten und nicht mit Fehlanreizen verbunden sind.

Autorenerklärung: Die Daten zu Diabetes mellitus entstammen Studien, die ohne inhaltliche Einflussnahme durch Sanofi-Aventis und Novo Nordisk sowie durch das Nationale Aktionsforum Diabetes mellitus im Rahmen von Drittmittelprojekten finanziert wurden.

\section{Literatur}

1 Glaeske G et al. Epidemiologische Methoden für die Versorgungsforschung. Gesundheitswesen 2009; 71: 685-693

2 Schubert I, Köster I. Diabetes mellitus: Versorgungsmonitoring auf der Basis von Routinedaten, In: Günster C, Klose K. Schmacke N, (Hrsg). Versorgungs-Report 2011. Stuttgart: Schattauer-Verlag: 2011: 129-144

3 Köster I, Huppertz E, Hauner H, Schubert I. Direct Costs of Diabetes Mellitus in Germany - CoDiM 2000-2007. Exp Clin Endocrinol Diabetes 2011; 119: $377-385$
I. Schubert

I. Köster

Qualitätsmanagement

Schlüsselwörter

GKV Routinedaten

$\checkmark$ Versorgungsforschung

Ondikatoren

Diabetes mellitus

Keywords

claims data

health service research

quality measures

diabetes mellitus

Institut

PMV forschungsgruppe, Klinik und Poliklinik für Kinder- und Jugendpsychiatrie, Universität zu Köln, Köln

Bibliografie

DOI $10.1055 / \mathrm{s}-0031-1286092$

Dtsch Med Wochenschr 2011;

136: S63 - (c) Georg Thieme

Verlag KG Stuttgart - New York . ISSN 0012-0472

\section{Korrespondenz}

\section{Dr. Ingrid Schubert}

PMV forschungsgruppe,

Klinik und Poliklinik für Kinderund Jugendpsychiatrie,

Universität zu Köln

Herderstraße 52

50931 Köln

Tel. 0221/478-6545

Fax 0221/478-6766

eMail

ingrid.schubert@uk-koeln.de 